\title{
Recomendações de atividade física para a saúde (pública): reflexões em busca de novos horizontes
}

\author{
Recommendations of physical activity for (public) health: \\ reflections in search of new horizons
}

Fabio Fortunato Brasil de Carvalho',2,3

${ }_{1}^{1}$ Programa de Pós-Graduação Stricto Sensu em Saúde Pública, Escola Nacional de Saúde Pública Sergio Arouca da Fundação Oswaldo Cruz (Ensp/Fiocruz) - Rio de Janeiro (RJ), Brasil

¿Área Técnica de Alimentação, Nutrição, Atividade Física e Câncer, Coordenação de Prevenção e Vigilância, Instituto Nacional de Câncer José Alencar Gomes da Silva (INCA)

- Rio de Janeiro (RJ), Brasil.

${ }^{3}$ Grupo de Trabalho de Promoção da Saúde e Desenvolvimento Sustentável, Associação Brasileira de Saúde Coletiva (ABRASCO) - Rio de Janeiro (RJ), Brasil.

DOI: https://dx.doi.org/10.7322/abcshs.v44i2.1199

\section{RESUMO}

Discutir a atividade física a partir de evidências que questionam as atuais recomendações de frequência, duração e intensidade para a saúde a partir da defesa de que foram incorretamente interpretadas e disseminadas, além de apontamentos sobre lacunas nas pesquisas que culminaram nas recomendações é o objetivo deste artigo. Foi realizada pesquisa na literatura para reunir elementos sobre os temas abordados e trazer luz a novas interpretações e compreensões, em um texto de caráter ensaístico. A partir da explicitação da complexidade envolvida na prática de atividade física, destacam-se os múltiplos fatores envolvidos e defende-se a construção de possibilidades que são negociadas e pactuadas com os sujeitos, assim como o apoio processual a partir daí. Em conclusão, novos horizontes são apontados mediante a apresentação de documentos institucionais com destaque para a necessária ampliação da compreensão da relação entre atividade física e saúde na qual extrapola-se o biológico, assim sua realização não deve atender simples e unicamente às recomendações.

Palavras-chave: Saúde pública; promoção da saúde; exercício.

\section{ABSTRACT}

To discuss physical activity based on evidence that questions the current recommendations of frequency, duration and intensity for health from the defense of which they were incorrectly interpreted and disseminated, besides notes about gaps in the research that culminated in the recommendations is the objective of this article. Literature research was carried out to gather elements on the topics covered and bring light to new interpretations and understandings, in an essay text. From the explanation of the complexity involved in the practice of physical activity, it is emphasized the multiple factors involved and defend the construction of possibilities that are negotiated and agreed with the subjects, as well as the procedural support from there. In conclusion, new horizons are pointed out through the presentation of institutional documents highlighting the necessary extension of the understanding of the relationship between physical activity and health in which the biological is extrapolated, so its realization should not only meet the recommendations.

Keywords: Public health; health promotion; exercice. 


\section{INTRODUÇÃO}

A atividade física (AF) tem amplo reconhecimento como ação de saúde individual e coletiva, em especial como fator de proteção para as doenças crônicas não transmissíveis (DCNT), em grande parte devido ao que foi denominado como discurso da vida ati$\mathrm{va}^{1,2}$, que buscou disseminar o componente informacional.

Benefícios provenientes da AF para a saúde estão documentados na literatura científica e encontram farta aceitação no senso comum. Os mais variados temas são relacionados desde a sua interpretação como fator de proteção relacionado à saúde, rivalizando com o risco ${ }^{3}$, qualidade de vida ${ }^{4,5}$, compreensões sobre o sedentarismo ${ }^{6}$, inserção do Professor/Profissional de Educação Física no Sistema Único de Saúde (SUS) ${ }^{7}$, entre outros.

Em 2017 ocorreram importantes 'movimentações' institucionais da Organização Mundial da Saúde $(\mathrm{OMS})^{8}$ e do Programa das Nações Unidas para o Desenvolvimento (PNUD) ${ }^{9}$ que evidenciaram a importância da AF para a saúde.

Atualmente a principal recomendação de AF para a saúde é a da Organização Mundial da Saúde (OMS) publicada em 2010, a qual será apresentada e debatida posteriormente, mas e se as evidências que embasaram as atuais recomendações de AF para a saúde estiverem sendo erroneamente interpretadas? A partir daí explorar-se-á algumas questões que poderão dar novos horizontes para a pesquisa, políticas públicas e programas, que são baseados em dados que podem estar incorretos já que as diretrizes de AF para a saúde pública dependeram de comparações individuais, em vez de uma avaliação sistemática da evidência disponível ${ }^{10}$.

Posteriormente debater-se-á questões como a complexidade envolvida na realização da $\mathrm{AF}$ e, em conclusão, serão feitos alguns apontamentos sobre o que se avizinha no horizonte sobre o tema. Assim, problematizar questões relacionadas à AF para a Saúde Pública tem relevância dada a aposta nela como medida de saúde individual e coletiva.

Para a confecção deste manuscrito, de caráter ensaístico, foi realizada pesquisa na literatura com caráter exploratório para reunir elementos sobre os temas abordados e trazer luz a novas interpretações. Além de outras leituras e reflexões sobre temas correlatos como a Promoção da Saúde.

\section{DISCUSSÃo}

\section{Recomendações de atividade física para a saúde}

$\mathrm{O}$ desenvolvimento e a publicação de recomendações de $\mathrm{AF}$ baseadas em evidências podem informar as políticas nacionais e outros tipos de intervenções em saúde pública, como as pesquisas e ser usadas por profissionais de saúde ${ }^{11}$. Logo, é possível inferir que se as recomendações estão sendo incorretamente interpretadas, toda a cadeia de produção e difusão de conhecimentos, além da AF como oferta de cuidado, também seriam atingidos.
Atualmente a maioria das diretrizes internacionais recomenda um objetivo de 150 minutos ( $\mathrm{min}$ ) de AF de intensidade moderada a vigorosa por semana, tal qual a OMS, que recomenda para adultos com idade superior a 18 anos a realização de pelo menos 150 min de atividade aeróbica de intensidade moderada, ou 75 min de intensidade vigorosa por semana, ou uma combinação de ambas. Afirma ainda que a AF aeróbia deve ser realizada em sessões de pelo menos 10 min de duração ${ }^{11}$.

Essa recomendação é reproduzida em instrumentos utilizados para a pesquisa como questionários de atividade física (GPAQ/ IPAQ), utilizados para vigilância de fatores de risco para DCNTs. No Brasil essa recomendação aparece em distintas publicações, inclusive direcionadas para a Atenção Básica, onde a AF é mais ofertada no SUS ${ }^{12}$, além de ser utilizada como parâmetro em grandes pesquisas nacionais como a Pesquisa Nacional de Saúde, o Vigitel e outras.

A partir da hipótese de que um volume muito inferior ao que é atualmente recomendado estaria associado a benefícios de saúde significativos e clinicamente relevantes, particularmente em indivíduos inativos e aqueles que vivem com condições médicas crônicas, afirma-se que nenhum limiar mínimo de AF existia para obter benefícios para a saúde ${ }^{12}$. Metade do volume e/ou intensidade recomendados por diferentes instituições da saúde pode conduzir a benefícios para a saúde, já que atingir a recomendação de 150 min de $\mathrm{AF}$ por semana pode não ser prático para grande parte da sociedade contemporânea ${ }^{13}$.

As adaptações orgânicas/fisiológicas da AF não atendem a um princípio objetivo, no sentido cartesiano, ou seja, tais adaptações não ocorreriam, por exemplo, se a AF de intensidade moderada for realizada por 29 min durante cinco dias na semana, totalizando $145 \mathrm{~min}$ ou ainda em três sessões diárias que totalizem $30 \mathrm{~min}$, divididos em dois de 9 e um de 12 min.

Ou ainda, a partir do risco como probabilidade ${ }^{6}$, quando a $\mathrm{AF}$ é realizada abaixo das recomendações não haveria alguma 'proteção', ainda que não o percentual estabelecido na literatura, já que a categorização de inativo fisicamente, em muitas pesquisas, é baseada nas indicações citadas anteriormente.

Dentre as recomendações, a OMS afirmou que o volume de AF varia de acordo com a prevenção das diferentes DCNTs, contudo ressaltou que a evidência científica era insuficientemente precisa para orientações separadas para cada condição de saúde específica, mas seria forte o suficiente para cobrir todos os resultados de saúde selecionados ${ }^{11}$. A formulação de uma recomendação única que proporcione efeitos benéficos para todos os desfechos da saúde ainda é um grande desafio para a ciência ${ }^{14}$.

Várias curvas dose-resposta podem existir e a melhor e a mínima dosagem provavelmente variam com base no resultado primário a medir. Pesquisa adicional é necessária para olhar para as várias relações dose-resposta de acordo com a condição 
de saúde. As recomendações de AF foram projetadas para otimizar benefícios à saúde em vez de fornecer dosagens mínimas, contudo foi o que ocorreu ${ }^{12}$.

Não há dúvidas que a participação da $\mathrm{AF}$ em nível consistente com as recomendações internacionais pode levar a benefícios significativos para a saúde e uma redução do risco de mortalidade prematura em pelo menos 25 condições de saúde, mas ressalta-se que há crescente evidência que este volume não é o nível mínimo exigido para benefícios de saúde e pode até mesmo estar mais perto do nível ótimo dependendo da condição de saúde ${ }^{12}$.

A ausência de consenso sobre a quantidade mínima de AF pode trazer repercussões negativas tanto no âmbito do cuidado, no qual sujeitos podem ser estimulados a manter uma quantidade insuficiente, seja ela qual for, quanto no âmbito das políticas e pesquisas. Quando a prevalência adequada na população apresenta resultados antagônicos e deixa dúvidas, pode haver prejuízos em metas e objetivos pactuados em programas e políticas de saúde pública ${ }^{14}$.

A partir de dados do Vigitel, verificou-se que mais da metade dos participantes foram classificados de modo discordante pelas principais recomendações mundiais de $\mathrm{AF}$, ou seja, quem praticou os mesmos minutos por semana (mesmo volume de AF) foram classificados de forma divergente, mostrando que ainda existem dúvidas a respeito da quantidade mínima necessária para alcançar benefícios para a saúde ${ }^{14}$.

Destaca-se que diferenças entre as recomendações podem ser explicadas, em parte, pelo interesse em diferentes desfechos como o efeito protetor da $\mathrm{AF}^{14}$. Tais disparidades foram apontadas a partir de limites teórico metodológicos dos estudos epidemiológicos relacionados, existindo imprecisão proporcionada por incongruências entre os valores das distintas medidas de sedentarismo ou inatividade física ${ }^{15}$.

Aborda-se, ainda, os benefícios da AF de intensidade leve, que também traz benefícios para a saúde e não são abordadas nas recomendações ${ }^{12,16}$. Atualmente, afirma-se que simplesmente mover-se mais conduz a benefícios significativos para a saúde, em especial a partir da leitura de que atingir a recomendação, para muitos indivíduos, pode não ser viável e distante criando um alvo inalcançável ${ }^{12}$.

Houve um erro na tradução do conhecimento que gerou a interpretação da existência de um limiar, ou seja, só haveria benefícios a partir da duração recomendada, uma espécie de 'tudo ou nada'12. Conclui-se que os resultados da pesquisa refutam diretamente as recomendações que implicam ou declaram explicitamente que é preciso atingir o limite de $150 \mathrm{~min}$ por semana de AF de intensidade moderada para obter benefícios de saúde ${ }^{12}$.

Utiliza-se o equivalente metabólico (MET) como uma das formas de determinar a intensidade da AF, trata-se de um "múltiplo da taxa metabólica basal, equivalente à energia suficiente para um indivíduo se manter em repouso, representado na literatura pelo consumo de oxigênio (VO2) de aproximadamente $3,5 \mathrm{ml} / \mathrm{kg} / \mathrm{min}^{\prime 17}$.

Logo, o número de METs significa quantas vezes o metabolismo de repouso foi multiplicado na AF, X METs significam que a intensidade foi de $\mathrm{X}$ vezes o gasto energético maior do que o de repouso ${ }^{17}$.

Em geral, a AF é classificada, quanto à intensidade, de acordo com compêndios existentes ${ }^{14}$, contudo questiona-se se é possível afirmar, a priori, a intensidade da AF considerando, entre outras coisas, as adaptações orgânicas/fisiológicas e o princípio da especificidade e individualidade. Destaca-se ainda que em compêndios a mesma modalidade ou tipo de AF pode variar em mais de $100 \%$, ou seja, o ciclismo pode ir de 4 a 12 METs, por exemplo, ou a corrida pode ir de 4,5 a 18 METs a depender da velocidade, se existe ou não aclive ou declive, faixas etárias, entre outros ${ }^{18,19,20,21}$.

Uma questão que merece ser observada, nas mensagens mais atuais a partir das recomendações, é que não aparece mais a predominância do metabolismo aeróbio da $\mathrm{AF}$, conforme consta nas recomendações da $\mathrm{OMS}^{11}$. Vale lembrar que nesta também havia a recomendação de $\mathrm{AF}$ relacionada à força e resistência muscular, mas elas geralmente não eram divulgadas vis a vis com a aeróbia.

Ora, por definição, atividades como alongamento e flexibilidade, por exemplo, também são uma possibilidade de AF e podem não alcançar os METs que a classificariam como AF de intensidade leve e assim trazer os benefícios para a saúde a partir dos mecanismos fisiológicos/orgânicos envolvidos, ainda que estes não estejam completamente elucidados, ao menos não é comum na literatura pesquisas que analisem a intensidade deste tipo de AF. Tal questão merece ser melhor explorada e opta-se por não fazê-lo neste momento.

Outra questão é que recomendações populacionais não atingem a especificidade individual de determinados sujeitos, ou seja, para um sujeito ter benefícios para a saúde pode ser necessário realizar mais ou menos AF, seja qual for a recomendação.

A medição da AF também é apontada como uma questão a ser considerada, há discrepâncias entre a duração e a intensidade autorrelatadas e as objetivamente medidas, superestimando a prática em até $50 \%$ ou mais ${ }^{12,13}$. As recomendações são formadas com base em um grande conjunto de evidências epidemiológicas que dependem quase exclusivamente de dados autorrelatados, sendo necessário reconhecer esta inconsistência ${ }^{12}$.

É desafiador validar a medição precisa da AF em sujeitos em seu cotidiano, pois as medidas objetivas possuem limitações como os custos financeiros, a inclinação do terreno, etc ${ }^{22,23}$ e os pesquisadores reconhecem uniformemente as limitações das informações autorrelatadas, mas isso raramente é traduzido para o público em geral ${ }^{10,12,13,22}$. 
Assim, o atual estágio de conhecimento que relaciona AF e saúde ainda apresenta importantes lacunas, o que não necessariamente invalida o atual estágio da pesquisa, produção e disseminação do conhecimento, mas é essencial reconhecê-las para tornar possíveis mais avanços a partir delas.

\section{Para além das recomendações}

As recomendações estão focadas nos níveis de AF para a prevenção de DCNTs, o que julga-se ser uma compreensão restrita de sua relação com a saúde ${ }^{16}$. A prática de AF normatizada dentro das recomendações institucionais perde seu caráter hedonístico e adota características da medicalização por considerá-la como um fenômeno puramente biológico e essencialmente determinante para um estado de saúde ideal e, assim, desconsidera ou coloca em um plano secundário os processos de relações sociais que contribuem para a sua prática ${ }^{24}$.

A AF é um comportamento complexo e multidimensional ${ }^{8,16,22,23}$, contudo ainda é hegemônica a compreensão de que esta prática depende apenas da vontade e esforço pessoal, desconsiderando diversos fatores que favorecem ou não a realização dentre eles a urbanização, segurança, instalações e programas públicos, condições de saúde, renda, ocupação, escolaridade....11,22,24,25,26,27,28 o que denota a importância do envolvimento de outros setores além da Saúde tais como a Educação, Transporte, Planejamento Urbano, entre outros ${ }^{8}$.

Para fins de pesquisa, a AF é dividida em domínios específicos: lazer (ou recreativo), ocupacional, doméstico e de deslocamento (transporte $)^{22}$. O domínio lazer é comumente considerado o mais medido e a principal forma modificável de realização da $\mathrm{AF}^{10,23}$.

A AF também é produto de como a sociedade pauta a vida coletiva, com as possibilidades de escolhas, acesso e usufruto dessas práticas sendo desigualmente distribuídas, com marcantes diferenças, por exemplo, por gênero ${ }^{9,16,29}$, a prevalência de inatividade física no lazer é maior entre o sexo feminino, idosos e nas faixas de menor escolaridade e renda ${ }^{24}$. Demais disparidades na AF estão no deslocamento e/ou ocupação de indivíduos com menor escolaridade, menor renda e/ou pior qualificação profissional, já no lazer, há maior nível em sujeitos com maior renda, maior escolaridade e com melhor status profissional ${ }^{24}$.

A partir dos fatores supracitados, compreender e difundir a AF como uma forma de corrigir e normalizar comportamentos, prescrevendo a realização com a finalidade única de não adoecer, ainda muito comum, inclusive por profissionais de saúde, é uma das hipóteses do afastamento de sujeitos e coletividades destas práticas. De forma geral, há a compreensão que a prática regular é fundamental para estar saudável, contudo a adesão ainda é muito baixa ${ }^{9}$.

Não se trata de hierarquizar ou relativizar os benefícios da AF, ou seja, acreditar que benefícios de uma ou outra ordem sejam mais importantes, mas de mostrar que há outros elementos além das recomendações. Defende-se que a AF deve fazer parte de formas de andar a vida com desejo, prazer, interesse, entre outros, que permanecem dentro de certos limites sensíveis que contribuam para a saúde $\mathrm{e}^{30,31}$.

A literatura aborda a possibilidade de programas de AF na comunidade incidirem, por exemplo, sobre os níveis de organização e engajamento com as questões da vizinhança ${ }^{25}$, há relatos de como a aproximação e a convivência, independente dos benefícios biológicos, são reconhecidas como questões-chave para a prática de AF em programas no SUS ${ }^{32,33}$, assim como o julgamento afetivo e o prazer também serem reconhecidos como preditores importantes para a adesão à $\mathrm{AF}^{12}$.

Em um exemplo a partir de um programa relacionado ao Setor de Esporte e Lazer, evidenciando a AF para além do Setor Saúde, afirma-se ser importante e legítimo buscar benefícios biológicos ou ainda direcionar ações para grupos que necessitam de cuidados específicos, no entanto, é preciso que se tenha uma visão ampliada contemplando os determinantes sociais que podem auxiliar ou atrapalhar a possibilidade dos sujeitos terem uma vida com maior ou menor qualidade ${ }^{29}$.

Já em programa municipal de AF no SUS, a análise permitiu afirmar que além de mitigar iniquidades relacionadas a essa prática, ele pode influenciar a população do entorno já que sua presença estimula a participação ${ }^{25}$. Defende-se que a AF seja planejada e ofertada de forma a incentivar ações cotidianas ampliem o potencial individual e coletivo para a efetivação de modos de vida mais saudáveis ${ }^{29}$.

Aqui é importante a apresentação de outra face da AF como ação de saúde individual e coletiva, que é a não desresponsabilizarão dos sujeitos ${ }^{22}$, com o que se concorda já que há também vontade, desejo, interesse pessoal e individual, na escolha, que pode ser mais ou menos livre, a depender dos contextos de vida. Não se trata da perspectiva culpabilizante quando sujeitos optarem pela não realização da $\mathrm{AF}$, ou ainda quando ela não for factível, mas de reconhecer possibilidades de sujeitos exercerem sua autonomia, tema caro para a vertente crítica da Promoção da Saúde, referencial com o qual se afilia.

Defende-se a utilização, como estratégia, a construção de possibilidades que são negociadas e pactuadas entre os sujeitos, profissionais, trabalhadores e serviços de saúde a partir da realidade concreta de vida daqueles, e não a prescrição de comportamentos saudáveis sem que sejam analisadas as reais possibilidades e interesses dos sujeitos ${ }^{34}$.

\section{Novos horizontes?}

A partir da crítica de que a literatura tem sido focada no nível de AF e no gasto energético, restringindo a pesquisa e compreensão sobre a AF relacionada à saúde, afirma-se que é hora de uma mudança de paradigma para uma compreensão mais ampla e, portanto, uma abordagem diferente para diretrizes, pesquisa e 
promoção da $\mathrm{AF}^{16}$. Defende-se ser necessária uma abordagem das recomendações a partir da compreensão de que a interface com a saúde ultrapassaria o gasto energético e a prevenção de doenças ${ }^{16}$.

Pesquisas disponíveis sugerem que uma ação de saúde pública global é necessária com iniciativas que envolvam escolas, política, planejamento, transporte, setor Saúde, empresas etc ${ }^{22}$. Recentemente dois importantes organismos internacionais realizaram movimentações tendo a AF como tema central: o PNUD e a OMS.

Através do Relatório já citado, o PNUD pressupõe que a AF tem o potencial de enriquecer a vida e ampliar a liberdade de escolha dos sujeitos e com isso advoga que os governos adotem políticas públicas condizentes com sua importância para o desenvolvimento humano?.

O documento descreve princípios orientadores para ações relacionadas à AF na perspectiva de não ser limitada por falta de recursos financeiros, de tempo disponível e/ou de oportunidades, sendo uma decisão livre e consciente, o que a caracterizaria como vetor de desenvolvimento humano, de ser uma ferramenta para melhorar a saúde e não somente o tratamento e a prevenção de doenças; da necessidade de adoção de medidas que aumentem a adesão à $\mathrm{AF}$, especialmente nos grupos menos favorecidos, dada a existência de iniquidades no acesso? .

Defende a adoção de uma compreensão ampliada da AF já que optar exclusivamente um ou outro viés, biológico ou cultural, não dá conta da complexidade do fenômeno, apenas o recorta em pedaços. Afirma que o movimento não ocorre baseado apenas no somatório de funções de órgãos fisiológicos, mas partem de sujeitos, que possuem desejos, emoções, vontades, uma história de vida individual e coletiva, que estão imersos em sentidos culturais, submetidos a posições sociais, assim quaisquer ações, pedagógica ou política, não só no campo da saúde, que ignorarem essa complexidade terá poucas chances de sucesso?.

Complementa, critica quando há consideração restrita da AF baseada apenas no gasto energético em algumas ações e, com isso, a rotulagem de sujeitos em ativos ou inativos, restringindo repertórios de prática e desconsiderando modos de viver e a forma como se organizam na sociedade. Além do enfoque prioritário em mensagens sobre adoecimento ou morte precoce pela ausência da $\mathrm{AF}$, defende adequar às singularidades dos sujeitos e grupos sociais, assim como valorizar a pertinência dos significados/sentidos construídos?.

Já o plano de ação global sobre atividade física 2018 - 2030 da OMS, analisado em uma versão preliminar, recebeu diversas contribuições ${ }^{9}$. O plano de ação, na versão que está sendo debatida, reconhece a natureza complexa da $\mathrm{AF}$ em diferentes contextos e destaca a influência significativa de determinantes socioculturais, econômicos e contextuais. Destaca que o custo de não atuar sobre a AF é alto com impactos negativos sobre o ambiente, desenvolvimento econômico, bem-estar da comunidade e realização de uma melhor qualidade de vida para todos ${ }^{8}$.
A versão atual do plano de ação é informada pelos seguintes princípios: Abordagem do curso de vida; Equidade; Empoderamento de povos, famílias e comunidades; Abordagem baseada nos direitos humanos; Cobertura de saúde universal; Coerência das políticas; Engajamento e parceria intersetorial para ação conjunta; e Prática baseada em evidências ${ }^{8}$.

Apresentam-se algumas contribuições feitas ao projeto de plano de ação global mediante consulta aberta on-line entre agosto e setembro de 2017, respectivamente do governo brasileiro por meio do Ministério da Saúde (MS) e de uma organização mundial sobre Promoção da Saúde.

A contribuição do MS foi sugerir que o documento enfatize a Segurança Pública como uma área chave para promover a $\mathrm{AF}$ em países de baixa e média renda, como o Brasil, sendo também importante para a redução das desigualdades na saúde. O que encontra respaldo no documento, que inclui a necessidade de ações políticas para apoiar caminhadas e ciclismo como parte das necessidades diárias e contribuir para vida pública, sendo acompanhadas de medidas para criar um ambiente seguro e saudável, necessitando de provisão apropriada de infraestrutura e regulamentos de trânsito para prevenir acidentes nas vias e estradas ${ }^{8}$.

Para o MS, o destaque à segurança urbana como um grande desafio para promover a $\mathrm{AF}$ em todos os segmentos da população baseia-se na relação entre a maior prevalência de inatividade física entre indivíduos com níveis educacionais mais baixos, identificada em pesquisas nacionais, e a violência e vulnerabilidade como uma barreira significativa ao uso de espaços públicos.

A União Internacional de Educação e Promoção da Saúde (UIPES) afirma que até o momento a AF foi subjugada como uma questão-chave para a Promoção da Saúde. Ressalta que se o plano for bem implementado, haverá contribuições importantes para, pelo menos, oito dos Objetivos de Desenvolvimento Sustentável alcançando benefícios além da saúde.

Elogia a necessária abordagem intersetorial, reconhece a importância da AF na Atenção Básica mediante a avaliação do paciente e provisão de aconselhamento e sugere a inclusão de que os fatores de inatividade física estão ligados a aspectos societários e ambientais, incluindo novas tecnologias, globalização e urbanização, que estão transformando como as pessoas vivem e trabalham.

O plano de ação foi publicado em 2018 e propõe: Criar sociedades mais ativas mediante mudanças de normas sociais e atitudes; Criar ambientes ativos por meio de acesso equitativo a ambientes seguros para a prática; Criar pessoas ativas mediante criação de programas e ações em contextos variados; e Criar sistemas ativos mediante liderança, governança e parcerias multissetoriais.

Acompanhar a operacionalização deste plano no Brasil, entre outras iniciativas e ações, permitirá avaliar se toda a complexidade envolvida na prática de AF está sendo considerada, para além dos níveis, gasto energético e prevenção de DCNTs, o que mostraria novos horizontes. 


\section{CONSIDERAÇÕES}

Da inquietação com as formas como as recomendações foram propagadas, do apelo que a AF tem na atualidade e do espraiamento e conexões com os mais variados temas, da concordância que benefícios biológicos são desejáveis e necessários, mas que há estreitamento e limitação importantes quando só estes estão presentes, seja no planejamento, discurso e/ou realização da AF, surgiu este texto que buscou trazer novos (e antigos) elementos presentes na relação entre AF e saúde.

Foram problematizadas lacunas ou limitações sobre o atual estado da arte da pesquisa e compreensão da AF para a saúde como os desafios na medição da AF e a partir delas o desenvolvimento das recomendações e, ainda que houvesse consenso, como aplicá-las na realidade concreta de sujeitos e coletivos dada a complexidade e multidimensionalidade relatadas.

A relação da $\mathrm{AF}$ com a saúde extrapola o biológico, o que não diminui sua importância, saúde também é evitar adoecer e, quando ocorre, atenuar as repercussões, logo, sejam as adaptações e benefícios ligados ao sistema cardiovascular, peso e composição corporal, entre outros, elas são importantes. Também, não só!

Assim, a questão não é descer do ônibus 1 ou 2 pontos (ou paradas) antes do trabalho, subir escadas ou 'mover-se!' simples e unicamente para alcançar as recomendações de duração e intensidade, para a saúde, X min por semana. É necessário buscar sentido para essa prática, experimentar, conhecer distintas modalidades e formas de AF, ou fazê-las se forem ressignificadas a partir da realidade concreta e houver concordância com elas.

As discussões das relações entre AF e saúde, a partir do olhar da Saúde Coletiva, ainda carecem de novas abordagens, reflexões, ideias, debates e compartilhamento de saberes e visões. Temas como iniquidades em saúde, desresponsabilização versus culpabilização dos sujeitos sempre estarão na ordem do dia, sendo uma necessidade constante, pois não há 'uma só saúde', inequívoca, irrefutável, dominante e hegemônica. As recentes 'movimentações' institucionais fortalecerão o escrutínio do tema e, nesse sentido, espera-se que este texto seja mais uma contribuição.

\section{AGRADECIMENTOS}

À Área Técnica de Alimentação, Nutrição, Atividade Física e Câncer / Conprev / INCA e à Renata Maciel Santos de Oliveira pelas contribuições na última versão do manuscrito.

\section{REFERÊNCIAS}

1. Fraga $A B$, Louro GL. Exercício da informação: governo dos corpos no mercado da vida ativa. Tese (Doutorado) - Universidade Federal do Rio Grande do Sul. Porto Alegre: 2005.

2. Ferreira MS. Navegar é preciso, viver não é preciso: risco no discurso da vida ativa. Motriz. 2015;15(2):349-57.

3. Matiello Júnior E, Gonçalves A, Martinez JFN. Superando riscos na atividade física relacionada à saúde. Movimento. 2008; 14(1):39-61

https://dx.doi.org/10.22456/1982-8918.3761

4. Dorneles G, Kloeckner A, Galvão SL, Colato AS, Ribeiro J, Peres A. Comparação da composição corporal, capacidade cardiorrespiratória e qualidade de vida de mulheres com sobrepeso e obesidade com presença ou ausência de estresse. Arq Mov. 2014;10(2)

5. Santos ALP, Simões AC. Educação física e qualidade de vida: reflexões e perspectivas. Saúde Soc. 2012;21(1):181-92.

6. Ferreira MS, Castiel LD, Cardoso MHA. Sedentarismo mata? Estudo dos comentários de leitores de um jornal brasileiro on-line. Saúde Soc. 2017;26(1):15-28. http://dx.doi.org/10.1590/s0104-12902017155705

7. Martinez JFN, Silva AM, Silva MS. As diretrizes do NASF e a presença do profissional em Educação Física. Motrivivência. 2014;26(42):222-37. https://dx.doi.org/10.5007/2175-8042.2014v26n42p207

8. World Health Organization (WHO). Global action plan to promote physical activity 2018-2030: more active people for a healthier world. Geneva: World Health Organization, 2018.
9. Programa das Nações Unidas para o Desenvolvimento (PNUD). Relatório Nacional de Desenvolvimento Humano do Brasil . Movimento é vida: atividades físicas e esportivas para todas as pessoas: 2017. Brasília: PNUD, 2017.

10. Liu L, Shi Y, Li T, Qin Q, Yin J, Pang S, et al. Leisure time physical activity and cancer risk: evaluation of the WHO's recommendation based on 126 high-quality epidemiological studies. Br J Sports Med. 2016;50(6):372-8 https://dx.doi.org/10.1136/bjsports-2015-094728

11. World Health Organization (WHO). Global recommendations on physical activity for health. Geneva: WHO, 2010.

12. Warburton DER, Bredin SSD. Health benefits of physical activity: a systematic review of current systematic reviews. Curr Opin Cardiol. 2017;32(5):541-56. https://dx.doi.org/10.1097/HCO.0000000000000437

13. Warburton DE, Bredin SS. Reflections on physical activity and health what should we recommend? Can J Cardiol. 2016;32(4):495-504 https://dx.doi.org/10.1016/j.cjca.2016.01.024

14. Lima DF, Levy RB, Luiz OC. Recomendações para atividade física e saúde: consensos, controvérsias e ambiguidades. Rev Panam Salud Publica. 2014;36(3):164-70.

15. Palma A, Vilaça M. O sedentarismo da epidemiologia. Rev Bras Ciênc Esporte. 2010;31(2):105-19.

16. Silva K, Garcia LM, Rabacow FM, Rezende LF, Sá TH. Physical activity as part of daily living: Moving beyond quantitative recommendations. Commentary. Prev Med. 2017;96:160-2. https://dx.doi.org/10.1016/j.ypmed.2016.11.004 
17. Coelho-Ravagnani CF, Melo FCL, Ravagnani FCP, Burini FHP, Burini RC. Estimativa do equivalente metabólico (MET) de um protocolo de exercícios físicos baseada na calorimetria indireta. Rev Bras Med Esporte. 2013;19(2):134-8. http://dx.doi.org/10.1590/S1517-86922013000200013

18. Ainsworth BE, Haskell WL, Leon AS, Jacobs DR Jr, Montoye HJ, Sallis JF, et al. Compendium of physical activities: classification of energy costs of human physical activities. Med Sci Sports Exerc. 1993;25(1):71-80 http://dx.doi.org/10.1249/00005768-199301000-00011

19. Ainsworth BE, Haskell WL, Herrmann SD, Meckes N, Bassett DR $\mathrm{Jr}$, Tudor-Locke C, et al. 2011 Compendium of physical activities: a second update of codes and MET values. Med Sci Sports Exerc. 2011;:43(8):1575-81.

http://dx.doi.org/10.1249/MSS.0b013e31821ece12

20. Butte NF, Watson KB, Ridley K, Zakeri IF, McMurray RG, Pfeiffer KA, et al. A Youth Compendium of Physical Activities: activity codes and metabolic intensities. Med Sci Sports Exerc. 2018;50(2):246-56.

http://dx.doi.org/10.1249/MSS.0000000000001430

21. Farinatti PTV. Apresentação de uma versão em português do compêndio de atividades físicas: uma contribuição aos pesquisadores e profissionais em fisiologia do exercício. Rev Bras Fisiol Exerc. 2003;2:177-208.

22. Leitzmann M, Powers $H$, Anderson AS, Scoccianti C, Berrino $\mathrm{F}$, Boutron-Ruault $\mathrm{MC}$, et al. European Code against Cancer 4th Edition: Physical activity and cancer. Cancer Epidemiol. 2015;39(Suppl 1):S46-55. http://dx.doi.org/10.1016/j.canep.2015.03.009

23. Li T, Wei S, Shi Y, Pang S, Qin Q, Yin J, et al. The dose-response effect of physical activity on cancer mortality: findings from 71 prospective cohort studies. $\mathrm{Br} J$ Sports Med. 2016; 50(6):339-45.

http://dx.doi.org/10.1136/bjsports-2015-094927

24. Rodrigues PF, Melo M, Assis M, Oliveira. Condições socioeconômicas e prática de atividades físicas em adultos e idosos: uma revisão sistemática. Rev Bras Ativ Fís Saúde. 2017;22(3):217-32.

https://doi.org/10.12820/rbafs.v.22n3p217-232
25. Fernandes AP, Andrade ACS, Costa DAS, Dias MAS, Malta DC, Caiaffa WT. Programa Academias da Saúde e a promoção da atividade física na cidade: a experiência de Belo Horizonte, MG, Brasil. Ciênc Saúde Coletiva. 2017;22(12):3903-14. http://dx.doi.org/10.1590/1413-812320172212.25282017

26. Project for Public Spaces (PPS). The case of Healthy Places: improving health outcomes through placemaking. Disponivel em: https://www.pps.org/wp-content/uploads/2016/12/HealthyPlaces-PPS.pdf. Acesso em: 27 set. 2018

27. Romieu I, Dossus L, Barquera S, Blottière HM, Franks PW, Gunter $\mathrm{M}$, et al. Energy balance and obesity: what are the main drivers? Cancer Causes Control. 2017;28(3): 247-58. http://dx.doi.org/10.1007/s10552-017-0869-z

28. Loch MR, Guerra PH. Bikes vs Car: análise crítica do documentário de Fredrik Gertten. Interface. 2017;21(62):725-9. http://dx.doi.org/10.1590/1807-57622016.0788

29. Goellner SV. Vida Saudável: é tempo de viver diferente. Porto Alegre: Centro de Memória do Esporte, 2017.

30. Mássimo EAL, Freitas MIF. Riscos para doenças crônicas não transmissíveis na ótica de participantes do Vigitel. Saúde Soc. 2014:23(2):651-63.

http://dx.doi.org/10.1590/S0104-12902014000200024

31. Karlsen MP, Villadsen K. Health promotion, governmentality and the challenges of theorizing pleasure and desire. Body Society. 2016;22(3):3-30.

https://dx.doi.org/10.1177/1357034X15616465

32. Carvalho FFB, Carvalho Y. Outros... lugares e modos de 'ocupação' da Educação Física na Saúde Coletiva/Saúde Pública. Pensar Prática. 2018;21(4):957-67. https://doi.org/10.5216/rpp.v21i4.51336

33. Caballero RMS. Pedagogia das vivências corporais: educação em saúde e culturas de corpo e movimento. Tese (Doutorado) Universidade Federal do Rio Grande do Sul. Porto Alegre: 2015.

34. Carvalho FFB, Cohen SC, Akerman M. Refletindo sobre o instituído na promoção da saúde para problematizar 'dogmas'. Saúde Debate. 2017:41(espe 3):265-76. http://dx.doi.org/10.1590/0103-11042017s320 